\title{
DUST EMISSION FROM STAR FORMING CLOUDS: \\ A PROGRESS REPORT
}

\author{
P.G. MEZGER, A. SIEVERS, R. ZYLKA
}

MPIfR bolometer group*

Max-Planck-Institut für Radioastronomie, Auf dem Hügel 69, 5300 Bonn 1

- Other members of the MPIfR bolometer group are: R. Chini, H.P. Gemünd, G. Haslam, E. Kreysa, R. Lemke and J. Wink.

\section{Dust emission and star formation: The working plan}

Model computations of protostellar evolution depend very strongly on the initial conditions: Fragmentation of massive cloud cores or coagulation of substellar condensations, the physical state of gas and dust (e.g. the formation of ice-mantles and grain coagulation), the presence of magnetic fields and its effect on gas and dust, and the formation of accretion disks as a consequence of an initial angular momentum of the protostellar condensation. The MPIfR bolometer group together with the molecular spectroscopists R. Mauersberger and T.L. Wilson have embarked on a program aimed at the exploration of the earliest evolutionary stages of high- and low-mass star formation. Here follows a brief progress report.

\subsection{DUST EMISSION AS A PROBE OF DENSE REGIONS OF INTERSTELLAR MATTER.}

In principle both isotopic molecular lines and dust emission can be used as probes of the ISM. In a recent review Mezger (1990) has discussed the merits and limitations of both methods. Measurements of dust emission yield the beam averaged surface brightness

$$
\mathrm{I}_{\nu}=\mathrm{S}_{\nu} / \mathrm{\Omega}_{\mathrm{A}}=\mathrm{B}_{\nu}\left(\mathrm{T}_{\mathrm{d}}\right)\left(1-\mathrm{e}^{-\tau_{\nu}}\right)
$$

Here $S_{\nu}$ is the flux density per beam solid angle $\Omega_{A}, T_{d}$ an appropriate average dust temperature, $\tau_{\nu}=\mathrm{N}_{\mathrm{H}} \sigma H$ the optical depth of the emitting dust layer, $\sigma_{\nu}^{H}$ the dust absorption cross section per $H$-atom and $N_{H}=$ $\mathrm{N}(\mathrm{H})+2 \mathrm{~N}\left(\mathrm{H}_{2}\right)$ the total hydrogen column density. If $\tau_{\nu}<<1$ and both $\sigma_{\nu}^{H}$ and $\mathrm{T}_{\mathrm{d}}$ are known the hydrogen column density can be derived from the relation

$$
N_{H}=\frac{1}{\sigma B} \frac{I_{\nu}}{B_{\nu}\left(T_{d}\right)}
$$


A parametrized representation of $\sigma_{L}^{H}(\lambda)$ and formulae to estimate dust temperatures, hydrogen column densities and masses are given in Appendix A of Mezger, Wink and Zylka (1990) and in the above mentioned review. We refer to these papers and summarize here briefly those results needed in the following discussion.

i) The dust absorption cross section in molecular clouds (MCs) and cloud cores can be approximated by

$$
\sigma H=\tau_{\nu} / N_{H}=\left(Z / Z_{\odot}\right) \quad b \begin{cases}710^{-21} \lambda_{\mu m}^{-2} & \lambda_{\mu m} \geqslant 100 \\ 710^{-22} \lambda_{\mu_{m}}^{-1.5} & 40 \leq \lambda_{\mu m} \leqslant 100\end{cases}
$$

Here $Z / Z_{\odot}$ is the relative metallicity and $\lambda_{\mu_{m}}$ is the wavelength in $\mu \mathrm{m}$. $b=1$ corresponds to the Draine and Lee (1984) dust model. For dust clouds of medium density $\left(\mathrm{n}_{\mathrm{H}} \leqslant 10^{5} \mathrm{~cm}^{-3}\right)$ and high density $\left(\mathrm{n}_{\mathrm{H}} \geqslant 10^{6} \mathrm{~cm}^{-3}\right.$ we recommend the use of $b=1.9$ and 3.4 , respectively.

ii) For clouds of medium density isotopic CO transitions and dust emission yield values of $N_{H}$ and $M_{H}$ which agree within a factor of -2 . However, in dense $\left(n_{H}>10^{6} \mathrm{~cm}^{-3}\right)$ and cold $\left(T_{d} \sim T_{g}<20-30 K\right)$ regions molecular transitions become unreliable tracers of column densities, probably because molecules freeze out and form ice mantles around refractory grain cores. Due to the formation of ice mantles the parameter in the above relation appears to increase to $b \leq 3.4$.

iii) Line contamination of broadband dust emission becomes important only at relatively high gas temperatures.

iv) Grain coagulation has been predicted and observed in circumstellar disks but may also occur in protostellar condensations. If grains coagulate and form compact particles of average size $\bar{a}<<100 \mu \mathrm{m}$ but large compared to NIR wavelengths, one expects little effects on the FIR/submm absorption cross section but a strong reduction of the extinction cross section in the wavelength range from the UV to the NIR. If, on the other hand, coagulated grains comprise a large volume fraction of empty space the dust cross section can be affected just in the opposite direction (Mathis and Whiffen, 1989) i.e. no change at optical/NIR wavelengths but an increase of the absorption cross section at FIR/mm wavelengths.

v) When observing dust emission from dense condensations the telescope beam usually samples both cold $(\sim 10-20 \mathrm{~K})$ and warm( $30-100 \mathrm{~K})$ dust. Since warm dust is in most cases the principal contributor to the total IR luminosity cold dust contributes in essence only to the $\mathrm{mm} / \mathrm{submm}$ part of the combined spectrum. Second to a model fit a spectral decomposition of the observed dust emission spectrum of the form

$$
\mathrm{S}_{\nu}=\sum_{\mathrm{i}} \mathrm{B}_{\nu}\left(\mathrm{T}_{\mathrm{d}, \mathrm{i}}\right)\left[1-\mathrm{e}^{-\tau} \tau_{, i}\right] \Omega_{\mathrm{s}, i}
$$

will usually yield meaningful (dust) mass averaged grain temperatures if a minimum of $\mathrm{i}=2$ for $\lambda>30 \mu \mathrm{m}$ and $\mathrm{i}=3$ for $\lambda>10 \mu \mathrm{m}$ components are used. In many cases high angular resolution observations are needed to determine the fit parameters $\tau_{\nu, i}$ and $\Omega_{s, i}$. 


\subsection{MAPPING OF DUST EMISSION}

For the observations discussed in the following sections we used the MPIfR ${ }^{3} \mathrm{He}$ cooled bolometer (Kreysa, 1985) with the 3m IRTF and the $2.2 \mathrm{~m}$ U. of Hawaii telescope (UHT) on Mauna Kea, Hawail, $\left(\lambda=1300 \mu \mathrm{m}, \theta_{A}=90 "\right.$; $\lambda=350 \mu \mathrm{m}, \theta_{A}=30^{\prime \prime}$ for the IRTF and $\lambda 1300 \mu \mathrm{m}, \theta_{A}=128^{\prime \prime}$ for the UHT) and with the $30 \mathrm{~m}$ MRT on Pico Veleta, southern Spain $\left(\lambda=1300 \mu \mathrm{m}, \theta_{\Lambda}=11 "\right.$ "; $\left.\lambda=870 \mu \mathrm{m}, \theta_{\Lambda}=7^{\prime \prime}\right)$.

\subsection{IS THERE A CHANCE TO DETECT INDIVIDUAL PROTOSTARS?}

Stellar masses in the galaxy range from $\sim 100 \mathrm{~m}_{0}$ to $\sim 0.1 \mathrm{~m}_{0}^{\prime}$. The present star formation rate is $-2-3 \mathrm{moyr}^{-1}$. What triggers -star formation on the one hand, and what prevents gravitationally unstable clouds to collapse on the other hand is not yet well understood. Bimodal star formation, as originally formulated by Güsten and Mezger (1983) to explain galactic abundance gradients, suggests induced formation of massive stars (m ${ }_{23 \mathrm{~m}_{0}}$ ) in GMCs in spiral arms and the central region of the Galaxy, and spontaneous formation of stars in the whole stellar mass range, occuring primarily in clouds in the interarm region.

In any case cold $\left(\mathrm{T}_{\mathrm{d}} \sim 10-20 \mathrm{~K}\right)$ condensations of gas and dust are expected to represent the first evolutionary stages of star formation. Combination of the point source sensitivity of $S_{1300} \geqslant 150 \mathrm{mJy}(\sim 3$ times the rms noise) of the $30 \mathrm{~m}$ MRT operated in the mapping mode with a scan velocity of $8 \% / \mathrm{min}$, with an earlier estimate of the number of isothermal protostars within $1 \mathrm{Kpc}$ (Mezger et al. 1988) leads us to conclude that most of the nearby MCs $\left(D_{K p c}-0.1-0.2\right)$ should contain protostars of low and medium mass. Most of these protostars together with about hundred high mass protostars should be detectable with the MRT in the mapping mode.

\subsection{THE WORKING PLAN}

Most star forming MCs have been mapped in isotopic transitions of CO and CS with low and intermediate angular resolution. Molecular transitions and $\mathrm{mm} / \mathrm{submm}$ dust emission yield comparable results for medium density clouds $\left(\mathrm{n}_{\mathrm{H}}<10^{3} \mathrm{~cm}^{-3}\right)$, easily detect gas outflow from PMS objects, but fail to detect high-density protostellar condensations. Our observing strategy is based on these considerations and the fact that focal plane chopping limits high angular resolution MRT and SEST maps to a maximum size of $\sim 8^{\prime} \times 6^{\prime}$. We use molecular emission as a guide line for the dust emission surveys.

\section{Regions of low-mass star formation}

\subsection{MYERS CLOUD CORES}

Myers and collaborators have - by means of molecular spectroscopy surveyed nearby dust clouds and have catalogued a number of condensations. (For a recent review see Benson and Myers, 1989). It has 
been suggested that "Myers cores" might represent the earliest stages of low-mass star formation. This suggestion appeared to be strengthened by the fact that a number of low-luminosity IRAS sources were observed close to the center of some of the cores. We have begun a survey of Myers cores with the MPIfR bolometer used at $\lambda 1300$ and $350 \mu \mathrm{m}$ with the IRTF and at $\lambda 1300 \mu \mathrm{m}$ with the UHT. Results of this survey can be summarized as follows (Chini et al., priv.comm.).

Table 1

Statistical summary of Mauna Kea Observations of Myers cores without associated IRAS sources

\begin{tabular}{rccccc}
$\lambda_{\mu \mathrm{m}}$ & $\boldsymbol{\theta}_{\mathrm{A}} / \operatorname{arcsec}$ & $N_{\text {Obs }}$ & $N_{\text {det }}$ & $S_{\min } / \mathrm{JY}_{Y}$ & $S_{\text {det }} / \mathrm{JY}_{Y}$ \\
\hline 350 & 30 & 22 & 6 & 0.5 & $1.8-8.5$ \\
1300 & $90(128)$ & 39 & 32 & 0.2 & $0.4-4.4$ \\
\hline
\end{tabular}

A survey (i.e. maps of size $\sim 3^{\prime} \times 2^{\prime}$ ) of the cores with positive detections and of a number of additional cores with associated IRAS sources was made at $\lambda 1300 \mu \mathrm{m}$. Compiled in Table 2 are the observed and derived characteristics of 10 of these cores which are located in the Taurus cloud at a distance of $140 \mathrm{pc}$. For a constant column density the hydrogen mass within the telescope aperture (expressed in Table 2 as linear size d) should decrease $\propto \mathrm{d}^{2}$. This is in rough agreement with the hydrogen masses derived for the Myers cores with different telescope apertures. There are especially no signs of a dense central core as would be expected to develop in the case of free-fall contraction of a protostar. The only exception is TMC-1A, which is the position of a low-luminosity IRAS source about $\sim 2.5$ west of TMC1 and which therefore is probably unrelated with TMC1. In the MRT map shown in Fig.1 TMC-1A appears as a slightly extended source of integrated flux density $S_{1300} \sim 330 \mathrm{mJy}$.

Table 2

Observed and derived characteristics of some Myers cores in the Taurus cloud which are not. associated with IRAS sources

\begin{tabular}{|c|c|c|c|c|c|c|c|c|c|c|}
\hline \multirow[b]{3}{*}{ Name } & \multirow[b]{3}{*}{$\alpha_{1950}$} & \multirow[b]{3}{*}{$\sigma 1950$} & \multicolumn{4}{|c|}{$\mathrm{s}_{y} / \mathrm{J}_{\mathrm{y}}$} & \multicolumn{4}{|c|}{$M_{H} / M_{\odot}$} \\
\hline & & & \multicolumn{2}{|c|}{$\lambda_{\mu \mathrm{m}}=1300$} & \multirow[b]{2}{*}{$11 "$} & \multirow{2}{*}{\begin{tabular}{|c|}
350 \\
$30 "$
\end{tabular}} & \multicolumn{3}{|c|}{$T_{d}=10 \mathrm{~K}$} & \multirow[b]{2}{*}{$2.3 E 16$} \\
\hline & & & $\theta_{A}=128 "$ & $90 "$ & & & $\mathrm{~d}=2.7 \mathrm{E} 17$ & $1.9 \mathrm{E} 17$ & $6.3 E 16$ & \\
\hline$L 1489$ & $04^{\mathrm{h}} 01^{\mathrm{m}_{4}} 455$ & $26 \cdot 10 \cdot 33: 0$ & 2.7 & & $<0.15$ & 2.4 & 3.4 & & 0.1 & $<0.2$ \\
\hline L1498 & 0750.0 & $\begin{array}{lll}25 & 02 & 13.0\end{array}$ & 1.2 & & " & 1.8 & 1.5 & & 0.1 & $<0.2$ \\
\hline L1495C & 1025.0 & $\begin{array}{lll}28 & 08 & 20.0\end{array}$ & 1.4 & & $"$ & $<0.5$ & 1.7 & & $<0.03$ & $<0.2$ \\
\hline L1495 & 1057.5 & $\begin{array}{lll}28 & 03 & 53.0\end{array}$ & 1.3 & & $"$ & $<0.5$ & 1.6 & & $<0.03$ & $<0.2$ \\
\hline L1495B & 1230.0 & $\begin{array}{lll}28 & 38 & 39.0\end{array}$ & 2.1 & & $"$ & $<0.5$ & 2.6 & & $<0.03$ & $<0.2$ \\
\hline L1506 & 1529.0 & 251210.0 & 1.5 & & $"$ & $<0.5$ & 1.9 & & $<0.03$ & $<0.2$ \\
\hline TMC-2 & 2943.0 & 241854.0 & & 1.4 & $"$ & 2.9 & & 1.7 & 0.2 & $<0.2$ \\
\hline TMC-1A & 3631.2 & 253556.0 & & 0.5 & 0.33 & $<0.5$ & & 0.6 & $<0.03$ & 0.4 \\
\hline TMC-1 & $38 \quad 42.0$ & $\begin{array}{lll}25 & 35 & 45.0\end{array}$ & & 1.0 & $<0.15$ & 2.2 & & 1.2 & 0.1 & $<0.2$ \\
\hline L1521D & 2442.5 & 261213.0 & & 1.2 & . & - & & 1.5 & - & $<0.2$ \\
\hline
\end{tabular}

Mean volume and column densities of the observed Myers cores are of order $\mathrm{n}_{\mathrm{H}} \sim 10^{5} \mathrm{~cm}^{-3}$ and $\mathrm{N}_{\mathrm{H}} \sim$ some $10^{23} \mathrm{~cm}^{-2}$. The cloud cores TMC1 and TMC2, although well investigated by molecular spectroscopy, could not be detected in $\lambda 1300 \mu \mathrm{m}$ dust emission. 


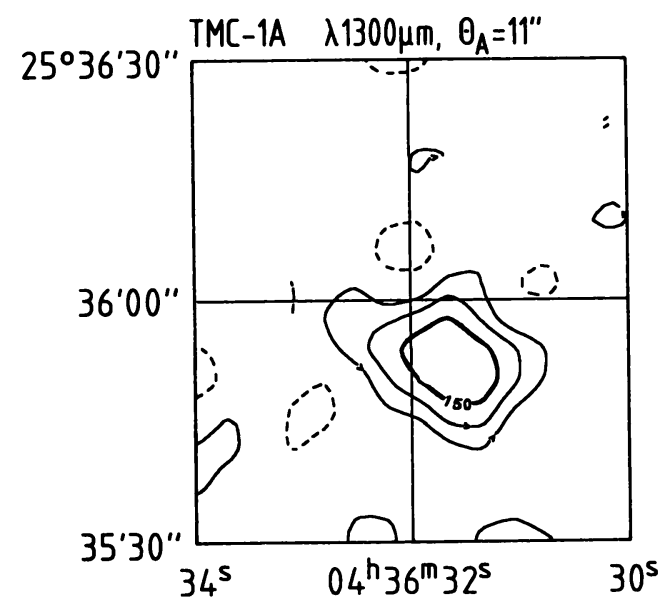

Fig.1.: MRT $\lambda 1300 \mu m$ map of TMC-1A. Contour levels are in mJy $\left(11^{\prime \prime}-\text { beam }\right)^{-1}$.

\subsection{LOW-MASS PMS OBJECTS}

Here we discuss three PMS objects which have been amply investigated and discussed in the literature. Dust emission from all three sources was observed at $\lambda 1.3 \mathrm{~mm}$ by Walker, Adams and Lada (1990, in the following referred to as WAL). We compare our average dust temperatures and associated gas masses with corresponding results by WAL to emphasize the importance of a multi-component analysis of the dust emission spectrum. WAL used a single-temperature, variable $m$ fit to their data. All these sources have been investigated in various molecular transitions but a detailed comparison with dust emission is outside the scope of this paper. IRAS 16293-2422 is a PMS object with gas outflow located in the Rho Ophiuchus cloud $\left(D_{\mathrm{Kpc}} \sim 0.16\right)$. WAL derive a flux density of $\mathrm{S}_{1300}=7.0 \mathrm{Jy}$. Although they do not resolve this source they observe emission out to $-60 "$. Their spectral fit yields $T_{d}=32 \mathrm{~K}, \mathrm{~m}=1.3$, an optical depth of $\tau_{1300}=3.0 \mathrm{E}-3$ and a gas mass of $\mathrm{M}_{H} \sim 2.0 \mathrm{~m}_{\odot}$. Mundy, Wilking and Myers (1986) resolved this source at $\lambda 2.7 \mathrm{~mm}$ into an elongated feature of size $11 " x<5$ " when convolved with the $4 " x 6 "$ beam.

Fig.2a shows the $\lambda 1300 \mu \mathrm{m}$ MRT image of this source. It consists of a central point source of FWHP 3" (not resolved with the MRT but derived from the spectral fit) with a flux density of $6.7 \mathrm{Jy}$ and an extended envelope of size $\sim 40 " \times 35^{\prime \prime}$ and flux density 7.6Jy. Fig.2b shows the spectrum fitted with a two component model. Note that the $\lambda 350 \mu \mathrm{m}$ flux density was obtained with a $30^{\prime \prime}$-beam in the ON-OFF mode so that part of the contribution from the envelope is suppressed and that the interferometer observations at $2.7 \mathrm{~mm}$ refer primarily to the central point source. In this fit the point source with $T_{d}=45 \mathrm{~K}$ and $\Omega_{s} \sim 10(\operatorname{arcsec})^{2}$ becomes opaque at $\lambda 1100 \mu \mathrm{m}$ and has a gas mass of $\mathrm{M}_{\mathrm{H}} \sim 1.5 \mathrm{~m}_{\odot}$. The cold $\left(T_{d} \sim 20 \mathrm{~K}\right)$ envelope has an optical depth of $\tau_{1300} \sim 1.2 \mathrm{E}-2$ and a gas mass of $M_{H} \sim 4.5 m_{\odot}$. (In both cases $b=3.4$ has been adopted). Assuming $a$ 
spherical density distribution one derives hydrogen volume and column densities for core and envelope of $n_{H}=510^{9} \mathrm{~cm}^{-3}, N_{H}=5.110^{25} \mathrm{~cm}^{-2}$ and $10^{7} \mathrm{~cm}^{-3}$ and $8.510^{23} \mathrm{~cm}^{2}$, respectively. Obviously one needs to know the core-halo structure for a correct interpretation of the observations.

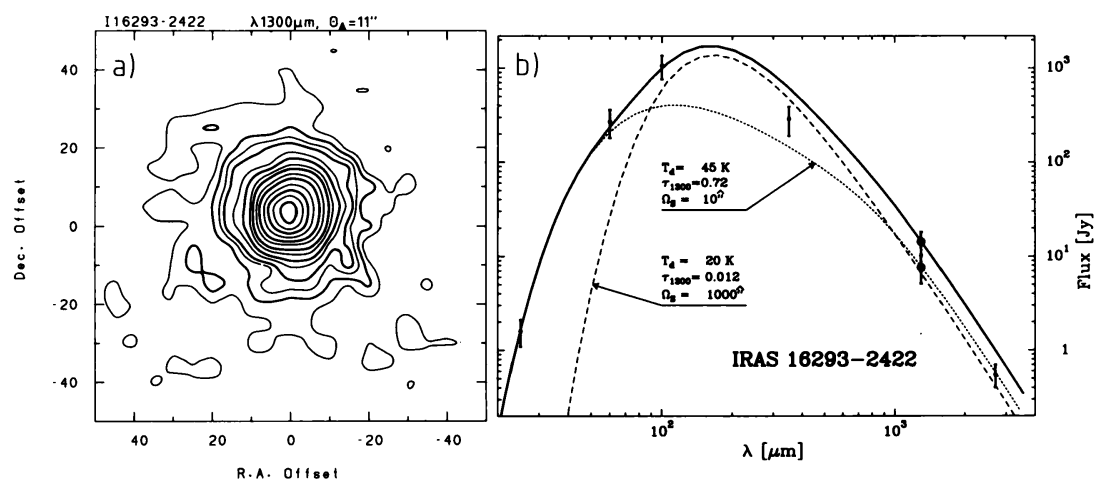

Fig.2.: IRAS 16293-2422. a) MRT $\lambda 1300 \mu m$ map. Coordinates are offset relative to the position $\alpha_{1950}=16^{h} 29^{20.9}, \delta_{1950}=-24^{\circ} 22^{\prime} 13^{\prime \prime}$. Contour levels are 100, 200, 300, 600, 800, 1100, 1400, 1900, 2400, 3100, 3800, 4500..mJy (11"-beam $)^{-1}$ b) Decomposed FIR/mm spectrum. Flux densities are from WAL and this paper ( 1300 and $350 \mu \mathrm{m}$ ).

L1551 IR5 is another outflow source at a distance $D_{K p c}-0.16$ for which WAL derive $T_{d}=48 \mathrm{~K}, m=1.2, T_{1300}=4.8 \mathrm{E}-4, L_{I R}=19.4 L_{0}$ and - with a flux density of $S_{1300}=4.3 \mathrm{Jy}-a$ total gas mass of $-0.6 \mathrm{~m}_{0}$. Their contour map shows a source of $\sim l^{\prime}$ extent with a fairly flat EW-plateau of emission at its center.

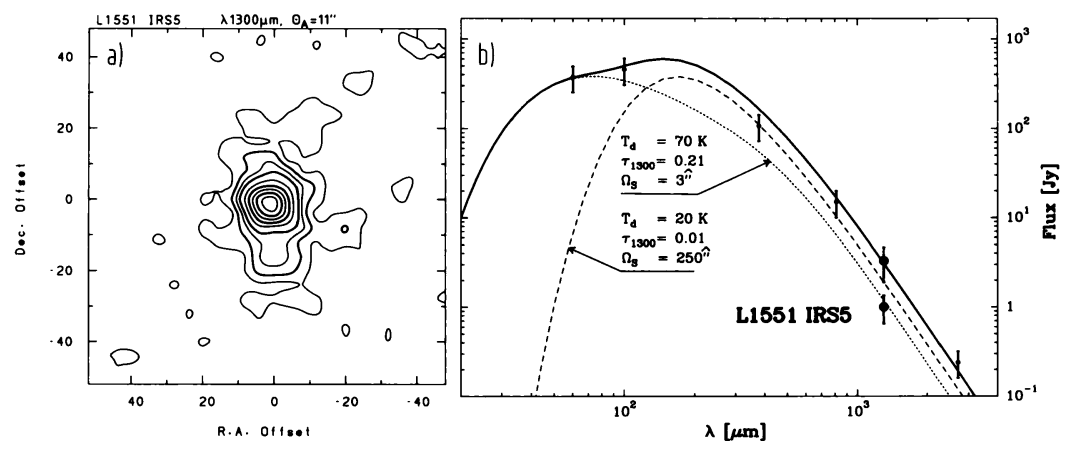

Fig.3.: L1551 IRS5. a) MRT $\lambda 1300 \mu m$ map. Coordinates are offset relative to the position $\alpha_{1950}=4^{h} 28^{m} 40.5, \delta_{1950}=18^{\circ} 1^{\prime} 42^{\prime \prime}$. Contour levels are 100, 200, 300, 400, 550, 700...mJy (11"-beam) ${ }^{-1}$. b) Decomposed FIR/mm spectrum. Flux densities are from WAL and this paper ( $11300 \mu \mathrm{m})$.

Fig.3a shows our MRT map. The structure is resolved into a point source of $\mathrm{S}_{1300} \sim 0.8 \mathrm{Jy}$ and an extended envelope of size $\sim 37^{\prime \prime} \times 20 "$ and flux density $2.5 \mathrm{Jy}$. The spectrum shown in Fig.3b requires again a 
two-component fit and yields source sizes of $\sim 2$ " for the point source (the MRT observations yield only an upper limit of the source diameter) and $\sim 16$ " for the envelope. Corresponding gas masses of core $\left(T_{d}=70 \mathrm{~K}\right)$ and envelope $\left(T_{d}=20 \mathrm{~K}\right)$ are (with $\left.b=3.4\right) \mathrm{M}_{H} \sim 0.1$ and $1.5 \mathrm{~m}_{\odot}$, respectively.

$\underline{B 335}$ is a large isolated dust globule at $D_{K p c} \sim 0.4$ with mass outflow and appears to have a stellar core of low luminosity (Keene et al., 1983). WAL. derive $\mathrm{T}_{\mathrm{d}}=25 \mathrm{~K}, \mathrm{~m}=1.1, \tau_{1300}=1.4 \mathrm{E}-3, \mathrm{~L}=8 \mathrm{~L}_{0}$ and with $\mathrm{S}_{1300}=0.8 \mathrm{Jy}$ a total gas mass of $1.1 \mathrm{~m}_{\odot}$. Actually, the spectrum of this source can be most simply interpreted in terms of a thin outer layer of warm dust $(\sim 19 \mathrm{~K})$ surrounding the interior cold $(\sim 14 \mathrm{~K})$ dust (Mezger, Mathis and Panagia, 1982). This is the typical dust temperature structure of a source which is mainly heated from outside by the general ISRF. A more recent spectral analysis confirms that the bulk of the emission comes from dust with $\sim 14 \mathrm{~K}$ (Gee et al., 1985). Fig.4 shows the $\lambda 1300 \mu \mathrm{m}$ MRT map where some spatial structure is indicated. We confirm the low $1300 \mu \mathrm{m}$ flux density of $0.8 \mathrm{Jy}$ observed by WAL. The corresponding mass is $M_{H} \sim 5 m_{\odot}$.

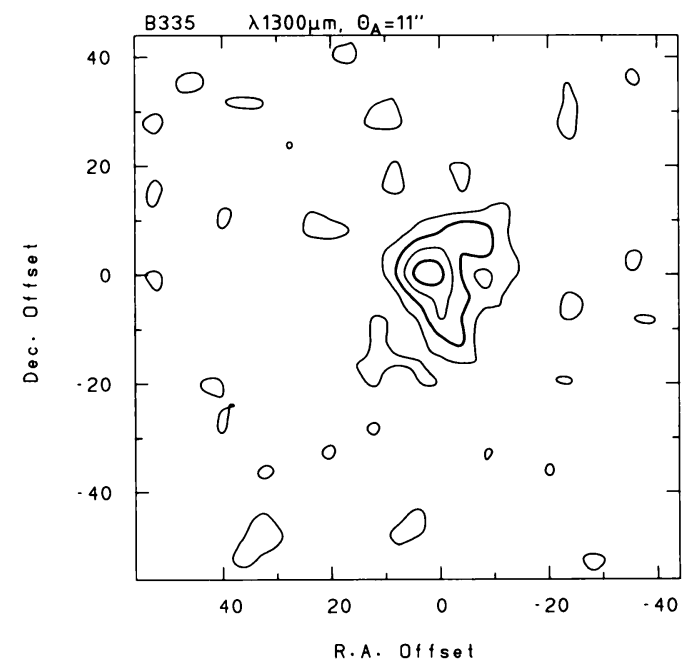

Fig.4.: B335. MRT $\lambda 1300 \mu \mathrm{m}$ map. Coordinates are offset relative to the position $\alpha_{1950}=19 h_{34} m_{34.7,} \delta_{1950}=$ $7^{\circ} 27^{\prime} 20^{\prime \prime}$. Contour levels are in $\mathrm{mJy}\left(15^{\prime \prime}-\text { beam }\right)^{-1}$.

\subsection{THE RO OPHIUCHUS CLOUD}

This cloud south of the star $\rho$ Oph is located at a distance of $160 \mathrm{pc}$ from the sun. It is considered to be the prototype of a low- to medium-mass star forming cloud. Wilking and Lada (1983) based on a $\mathrm{C}^{18} \mathrm{O}$ survey detected a $1 \times 2 \mathrm{pc}$ ridge with hydrogen column densities as high as $10^{23} \mathrm{~cm}^{-2}$, which extends from $\mathrm{SE}$ to $\mathrm{NW}$. It contains a total mass of $\mathrm{M}_{\mathrm{H}} \sim 400-500 \mathrm{~m}_{\odot}$. The internal structure of this cloud has been thoroughly investigated by means of molecular spectroscopy. Loren (1989) has described 89 dense clumps of which 12 very dense cores are claimed to be active sites of star formation. Wooten (1989) in a recent survey gives a very vivid but also somewhat gloomy description of this work, from which we quote: "that indeed the perception of the structure of dense cores depends critically upon the molecular transition in whose light it is imaged." 
In an attempt to detect low-mass isothermal protostars we began a survey of the $\lambda 1300 \mu \mathrm{m}$ dust emission from this cloud using both the IRTF and MRT. With the IRTF we observed at $\lambda 1300 \mu \mathrm{m}$ a cross section in $\alpha, \delta$ through the point $\alpha_{1950}=16^{\mathrm{h}_{2} 3^{\mathrm{m}} 25587, \delta_{1950}=-24^{\circ} 17^{\prime} 20^{\prime \prime} .7}$ and found extended emission ranging from $\sim 1$ to $10 \mathrm{Jy}\left(90^{\prime \prime}-\text { beam }\right)^{-1}$. This corresponds for $T_{d} \sim 15 \mathrm{~K}$ to hydrogen column densities ranging from $\mathrm{N}_{\mathrm{H}} \sim 2$ to $2010^{22} \mathrm{~cm}^{-2}$ and tends to confirm the existence of the above mentioned ridge structure. A number of MRT maps ranging in size from $3^{\prime} \times 2^{\prime}$ to $6^{\prime} \times 5^{\prime}$ were observed around peak positions of molecular line emission and some complex dust emission structure was indeed detected. One such structure is a submm source recently investigated at $\lambda 1100,800$ and $350 \mu \mathrm{m}$ with the UKIRT $3.8 \mathrm{~m}$ telescope by Ward-Thompson et al. (1989) and referred to as SM1. This source lies $\Delta \alpha \sim 456$ west and $33^{\prime \prime}$ south of a previsouly known FIR source observed by both Harvey, Campbell and Hoffmann (1979) and by IRAS which is referred to as IRS1. Ward-Thompson et al. derive from fits to the dust emission spectra of SM1 and IRS1 longward of $\lambda 25 \mu \mathrm{m}$ dust characteristics of $T_{d}=15 K, m \sim 2.2$ and $T_{d}=36 \mathrm{~K}, \mathrm{~m} \sim 1.0$. Their luminosities are $L_{I R} \sim 60$ and $110 L_{0}$.

Fig.5 shows the MRT map of SM1. It containes three condensations in an extended NS ridge. Flux densities are: $S_{1300} \sim 40 \mathrm{Jy}$ within the $150 \mathrm{mJy} /$ beam contour and $\sim 16 \mathrm{Jy}$ within the ridge structure defined by the $450 \mathrm{mJy} /$ beam contour. The condensations have flux densities of (from $\mathrm{N}$ to S) $4.5,6.5$ and $3.9 \mathrm{Jy}$. For $\mathrm{T}_{d}=15 \mathrm{~K}$ and $\mathrm{b}=1.9$ the total mass contained in the map is $\sim 60 \mathrm{~m}_{\odot}$; masses of the condensations range (for $b=3.4$ ) from -3 to $5.5 \mathrm{~m}_{\odot}$. Based on both the low dust temperature $\left(\mathrm{T}_{\mathrm{d}} \sim 15 \mathrm{~K}\right)$ and luminosity $\left(\mathrm{L}_{I R} \sim 60 \mathrm{~L}_{\odot}\right.$ ) we conclude that the three condensations represent isothermal low-mass protostars. The FIR source IRS1, on the other hand, appears to be a self-luminous PMS star embedded in a dust shell. The relatively low luminosity of $\mathrm{L}_{I R} \sim 110 \mathrm{~m}_{\odot}$ suggests a medium mass star as heating source.

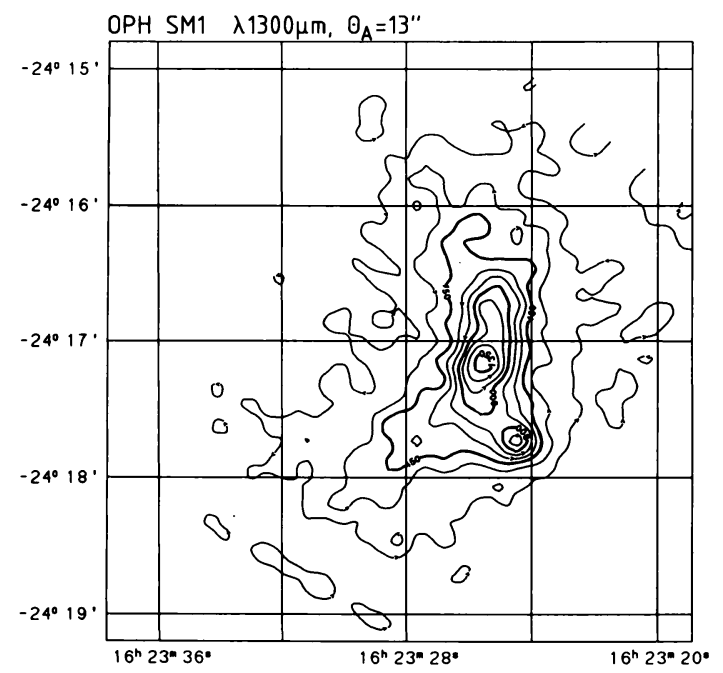

Fig.5.: $\quad$ MRT $\lambda 1300 \mu m$ map of the submm source SM1 in the pOph cloud. Contours are in $\operatorname{mJy}\left(13^{\prime \prime} \text {-beam }\right)^{-1}$. 
We also surveyed regions in $\rho$ Oph $B$ where Loren et al. (1980) and Martin-Pintado et al. (1983) had detected strong $\lambda 2 \mathrm{~cm} \mathrm{H}_{2} \mathrm{CO}$ emission which indicates the presence of cloud cores and condensations with volume and column densities of $\$ 10^{6} \mathrm{~cm}^{-3}$ and $\sim 10^{23} \mathrm{~cm}^{-2}$, respectively. Fig. 6 shows a contour map of $\lambda 1300 \mu \mathrm{m}$ dust emission from this region. An elongated structure with a surface brightness of $\sim 200 \mathrm{mJy}$ in a 15 " beam is visible, which corresponds for $T_{d}=15 \mathrm{~K}$ and $b=3.4$ to a hydrogen column density of $\mathrm{N}_{\mathrm{H}} \sim 1.510^{23} \mathrm{~cm}^{-2}$, in good agreement with the $\mathrm{H}_{2} \mathrm{CO}$ results. The total mass within the $200 \mathrm{mJy}$ per beam contour is $\sim 5 \mathrm{~m}_{\odot}$. Within this area there are no indications of condensations in the mass range $M_{H} \geq 0.3 m_{\odot}$.

\section{Regions of spontaneous high-mass star formation}

The Orion GMC is the region closest to the sun with (probably) spontaneous high-mass star formation. Two of its most prominent features are the Orion A and B clouds each of which contain some $10^{5} \mathrm{~m}_{\odot}$ of gas. Observations of dust emission from two cores in these clouds which contain $\sim 1000 \mathrm{~m}_{\odot}$ of gas have been published previously (NGC2024: Mezger et al., 1988; Mezger, 1988. OMC1/2, Mezger, Wink and Zylka, 1990). Embedded in these cores are massive isothermal protostars (NGC2024) and PMS objects (OMC1). Derived characteristics of cloud cores and dense condensations are given in Table 3. Fig.7a and $b$ show unpublished MRT $\lambda 870 \mu \mathrm{m}$ maps of the dense protostellar condensations - where free-free contamination is negligible - which fully confirm the morphology of the published $\lambda 1300 \mu \mathrm{m}$ maps.

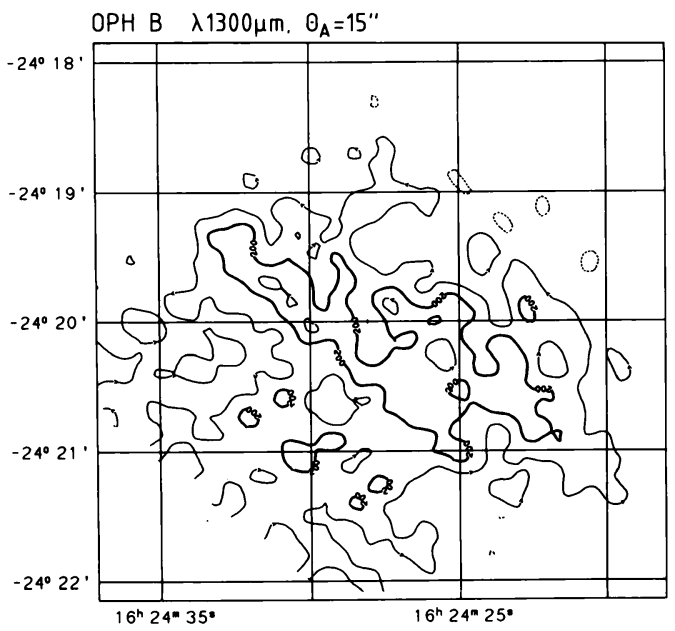

Fig.6.: $\quad$ MRT $\lambda 1300 \mu m$ map of the cloud core $\rho O p h \quad B$. Contours $\operatorname{mJy}\left(15^{\prime \prime}-\text { beam }\right)^{-1}$.

A review of the wealth of more recent observations of the NGC2024 cloud core is beyond the scope of this paper. Here we just want to point out some problems in interpreting limited data of a very complex region of the ISM. 
Dust and gas temperatures: Moore et al.(1989) made a single fit to the dust emission spectrum with a modified Planck function $B_{\nu}\left(T_{d}\right) \nu^{m}$ and $\mathrm{T}_{\mathrm{d}}=47 \mathrm{~K}, \mathrm{~m}=1.6$. Since opaque dust with $16 \mathrm{~K}$ emits in essence only at wavelengths $\lambda \geqslant 400 \mu \mathrm{m}$ it is clear that the composite spectrum can be represented by dust with an opacity spectrum less steep than $m \sim 2$. Such a fit ignores, however, the fact that warm dust, represented by $\lambda 60 \mu \mathrm{m}$ emission, outlines the HII region (which is extended in RA) while cold dust, represented by $\lambda 1300 \mu \mathrm{m}$ mission, outlines the molecular line emission (which is extended in DEC).

Table 3

Physical conditions in the cloud cores NGC2024 and OMC1.

\begin{tabular}{|c|c|c|c|c|c|}
\hline Source & $\mathrm{n}_{\mathrm{H}} / \mathrm{cm}^{-3}$ & $\mathrm{~N}_{\mathrm{H}} / \mathrm{cm}^{-2}$ & $M_{H} / m_{\odot}$ & $T_{d} / K$ & Ref \\
\hline $\begin{array}{l}\text { NGC2024 core } \\
\text { isoth. protostars }\end{array}$ & $\begin{array}{l}210^{5} \\
4-1410^{8}\end{array}$ & $\begin{array}{l}310^{23} \\
2-610^{25}\end{array}$ & $\begin{array}{c}800 \\
10-60\end{array}$ & $\begin{array}{l}16 \\
16\end{array}$ & 1) \\
\hline $\begin{array}{l}\text { OMC1 } \\
\text { hot core=FIR2 }\end{array}$ & $\begin{array}{ll}10^{5} & \\
3-9 & 10^{7}\end{array}$ & $\begin{array}{l}210^{23} \\
10^{25}\end{array}$ & $\begin{array}{c}1700 \\
50\end{array}$ & $\begin{array}{ll}25 & \\
1-2 & 10^{2}\end{array}$ & 2) \\
\hline
\end{tabular}

1) Mezger et al., 1988

2) Mezger, Wink and Zylka, 1990

Mezger et al. (1988; see their Fig.8) noted that the cold and dense condensations which are clearly discernible in the high-resolution dust emission maps are not well traced by various molecular transitions. Wilson, Mauersberger and Mezger (in prep.) using the MRT have recently mapped the NGC2024 cloud core in rare $\mathrm{CO}$ and $\mathrm{CS}$ isotopes $\left(\mathrm{C}^{18} 0 \mathrm{~J}=2-1\right.$ and $\left.1-0 ; \mathrm{C}^{17} 0 \mathrm{~J}=2-1 ; \mathrm{C}^{34} \mathrm{~S} \mathrm{~J}=3-2\right)$. Molecular emission follows in general the dust emission but maxima in the line emission tend to coincide with dust emission minima. Such an anticorrelation of dust and molecular emission has been also found in the case of OMCl (Mezger, Wink and Zylka, 1990).

\section{4. $\mathbf{W 9}$ as an example of induced high-mass star formation}

We have mapped a number of dust clouds associated with giant HII regions which are probably located in main spiral arms and thus should be typical for induced high-mass star formation. Here we will discuss some preliminary observations of $\mathrm{W} 49$ which is located at a distance of $\sim 14 \mathrm{Kpc}$ (Sievers et al., in prep.).

Fig.8a shows a $\lambda 1300 \mu \mathrm{m}$ map obtained with the IRTF. This map is not corrected for free-free emission which accounts for $-36 \%$ of the integrated flux density of $\sim 113 \mathrm{Jy}$. A map obtained at the same wavelength with the MRT containes $\sim 26 \mathrm{Jy}$ after correction for free-free emission using the $\lambda 3 \mathrm{~mm}$ maps of Salter et al. (1989). This is about one third of the dust emission contained in the IRTF map. Fig.8b shows a MRT map at $\lambda 870 \mu \mathrm{m}$, where contamination by free-free emission is no longer a problem. Fig.8c shows the spectral index $r(870 / 1300 \mu \mathrm{m})$ of the surface brightness of dust emission. This spectral index has been derived by comparing $870 / 1300 \mu \mathrm{m}$ MRT maps convolved to the angular resolution of 
29".5, with which free-free emission has been measured (Salter et al., 1989) and which has been subtracted from the shorter wavelength maps.

NGC $2024 \quad 1870 \mu \mathrm{m} \quad \theta_{A}=11 " \quad$ OMC 1 1 $1870 \mu \mathrm{m} \quad \theta_{A}=11 "$
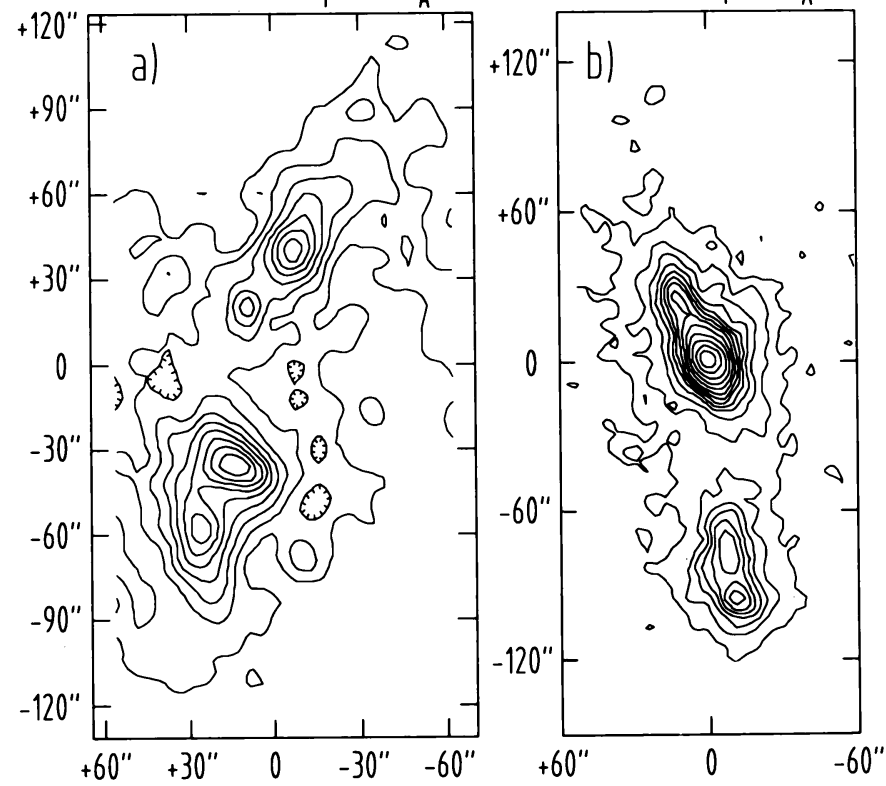

Fig.7.: MRT $\lambda 870 \mu m$ dust emission maps of NGC2024 and OMC1 Gauss-convolved to 11 arcsec (unpublished). Coordinates are offset relative to the position $\alpha=05^{h} 39^{m} 12^{5} .0, \sigma=-01^{\circ} 56^{\prime} 30^{\prime \prime}$ (NGC2024) and $05^{h} 32^{m} 46.9, \sigma=-05^{\circ} 24^{\prime} 26^{\prime \prime}(O M C 1)$.

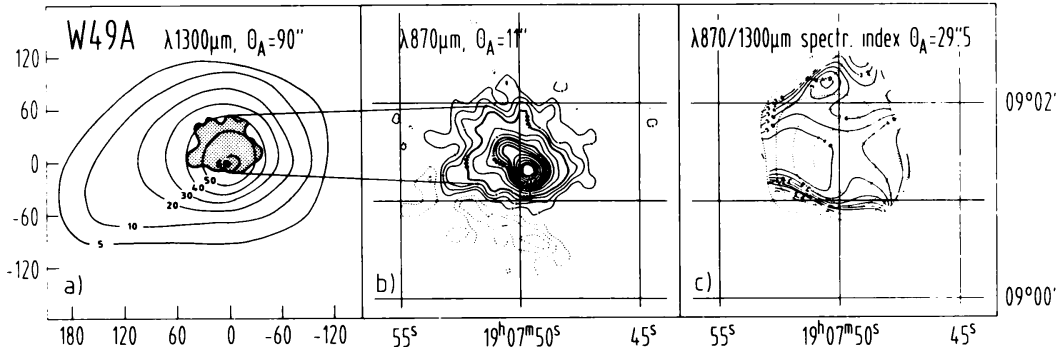

Fig.8.: The GMC associated with the Giant HII region $W 49$ a) IRTF $\lambda 1300 \mu \mathrm{m}$ map not corrected for free-free emission. The third-lowest contour of Fig.b) comprises the stipled area. Contours are in Jy $\left(90^{\prime \prime}-\text { beam }\right)^{-1}$ b) Gauss-convolved MRT $\lambda 870 \mu m$ map. Contours are in mJy $\left(11^{\prime \prime} \text {-beam }\right)^{-1}$ c) Contour map representation of the spectral index $r(870 / 1300 \mu \mathrm{m})$ derived from $M R T$ maps at $\lambda 1300$ and $870 \mu \mathrm{m}$, which are Gauss convolved to a FWHP of $\theta_{A}=29.5$ and from which free-free emission is subtracted. 
Over most of the central region of the map the surface brightness has a spectral index of $r^{\sim 4}$, as is expected for an opacity law $\sigma \not(\lambda) \propto \lambda^{-2}$ and temperatures $T_{d} \geqslant 50 \mathrm{~K}$. Towards the edge of the cloud the spectral index appears to decrease indicating the presence of dust with lower temperatures. This is in agreement with a decomposition of the submm/FIR/MIR spectrum shown in Fig.9 into three components with $\mathrm{T}-30$, 53 and $140 \mathrm{~K}$, respectively. Characteristics of these three components are given in Table 4. Note that the hydrogen masses derived from the luminosities of the three components are systematically lower than those derived from $\lambda 1300 \mu \mathrm{m}$ flux densities. This is due to opacity of the sources in the wavelength range of maximum emission. The extent of $30 \mathrm{~K}$ and $53 \mathrm{~K}$ dust emission is approximately outlined in Fig.8a by the 0.2 contour of the IRTF map and the second lowest contour of the MRT map, respectively.

Table 4

Observed and derived characteristics of "warm" GMC associated with W49.

\begin{tabular}{|c|c|c|c|c|c|c|c|c|}
\hline$\frac{T_{d}}{K}$ & $\frac{S_{1300}}{J y}$ & $\frac{\underline{M}_{H}{ }^{a)}}{m_{0}}$ & $\frac{\mathrm{n}_{\mathrm{H}}}{\mathrm{cm}^{-3}}$ & $\frac{\text { size }}{\mathrm{pc}}$ & $\frac{\mathrm{N}_{\mathrm{H}}}{\mathrm{cm}^{-2}}$ & $\bar{L}_{0}{ }^{L_{1}}$ & Rin8 & $\frac{\underline{\underline{M}}_{\mathrm{H}}{ }^{b}}{\mathrm{~m}_{\Theta}{ }^{\prime}}$ \\
\hline 30 & 46 & $2.2 \mathrm{E5}$ & $-10^{4}$ & $\sim 11$ & -3E23) & & $\sim 20$ & $1.0 \mathrm{E5}$ \\
\hline 53 & 26 & $6.6 \mathrm{E} 4$ & $\sim 10^{5}$ & $\sim 3.7$ & $\sim 1 E 24$ & $310^{7}$ & $\sim 60$ & 1. 3E4 \\
\hline 140 & $\sim 0.2$ & $\sim 1.7 \mathrm{E} 2$ & -20 & - & - & & $\sim 20$ & $2.8 \mathrm{E} 1$ \\
\hline
\end{tabular}

a) derived from the flux density at $\lambda 1300 \mathrm{~mm}$ and $b=1.9$

b) derived from the luminosity of the individual components and the dust luminosity-to-hydrogen mass ration computed for $T_{d}$.

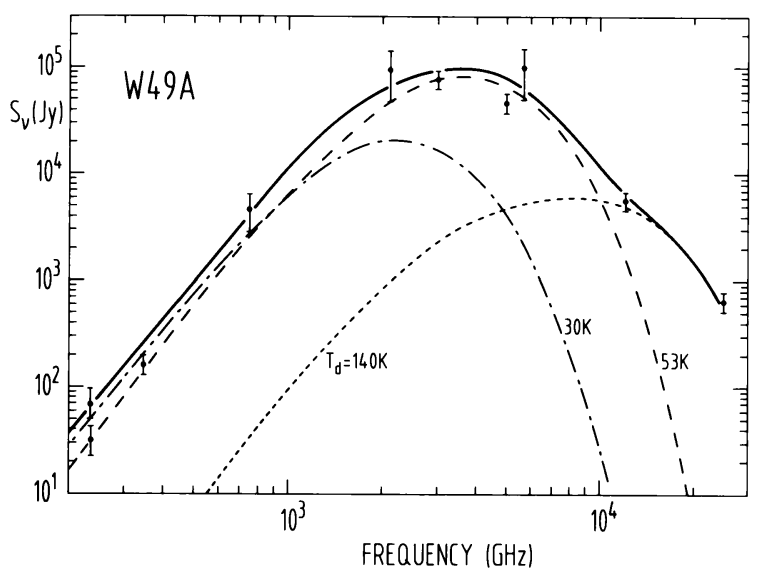

Fig.9.: Decomposed FIR/submm spectrum of W49. Characteristics of the components are given in Table 4. 


\section{References}

Benson, D.J., Myers, P.C.: 1989, ApJ. Suppl. 71, 89

Draine. B.T., Lee, H.M.: 1984, ApJ. 285, 89

Gee, G., Griffin, M.J., Cunningham, Ch.T., Emerson, J.P., Ade, P.A.R.:

1985, MNRAS 215, 15p

Güsten, R., Mezger, P.G.: 1983, Vistas in Astronomy 26, 159

Harvey, P.M., Campbell, M.F., Hoffmann, W.F.: 1979, ApJ. 228, 445

Keene, J. Davidson, J.A., Harper, D.A., Hildebrand, R.H., Loewenstein, R.F., Low, F.J.,Pernie, R.: 1983, ApJ. 274, L43

Kreysa, E.: 1985 in Int. Symp. on "mm- and submm wave Astronomy" URSI, Granada, p.153

Loren, R.B., Wooten, A., Sandquist, A., Bernes, C.: 1980, ApJ. 240, L169

Loren, R.: 1989, ApJ. 338, 902

Martin-Pintado, J., Wilson, T.L., Gardner, F.F., Henkel, C.: 1983, A\&A 117, 145

Mathis, J.S., Whiffen, G.: 1989, ApJ. 341, 808

Mezger, P.G., Mathis, J.S., Panagia, N.: 1982, A\&A 105, 372

Mezger, P.G., Chini, R., Kreysa, E., Wink, J.E., Salter, C.J.: 1988, A\&A 191,44

Mezger, P.G., Zylka, R., Salter, C.J., Wink, J.E., Chini, R., Kreysa, E., Tuffs, R.: 1989, A\&A 209, 337

Mezger, P.G.: 1988 in "Galactic and Extragalactic Star Formation" NATO-ASI Series 232, Pudritz and Fich, eds. p.227

Mezger, P.G., Wink, J.E., Zylka, R.: 1990, A\&A 228, 95

Mezger, P.G.: 1990 in Conf.Proc. "Physics and Composition of the Interstellar matter", Torun, Poland (in press)

Moore, T.J.T., Chandler, C.J., Gear, W.K., Mountain, C.M.: 1989. MNRAS 237, 1p

Mundy, L.G., Wilking, B.A., Myers, P.C.: 1986, ApJ 311, L75

Salter, C.J., Emerson, D.T., Steppe, H., Thum, C.: 1989, A\&A 225, 167

Walker, C.K., Adams, F.C., Lada, C.J.: 1990, ApJ. 349, 515 (WAL)

Ward-Tomson, P., Robson, E.I., Whittet, D.G.B., Gordon, M.A., Walther, D.M., Duncan, W.D.: 1989 , MNRAS 241, 119

Wilking, B.A., Lada, Ch.J.: 1983, ApJ. 274, 698

Wooten, A.: 1989, Proc. IAU Coll.No.120, eds. Tenorro-Tagle, Mole, Melznick, Lect.Notes in Phys. 350, p.210 